\title{
La bosinada nella letteratura dialettale di Milano
}

Allegrament l'è ch'il Bosin

Che fa di rim in Meneghin;

Semper provist de donitaa

Part Bamban, part veritaa

Don la mia cent ne foo l'invid

A senti qui che pang e qui che rid. ${ }^{1}$

[Eccovi l'allegro bosin

Che fa le rime in meneghino;

Fatte sempre con garbo

in parte frottola, in parte verità

Da lontano ci invita

A sentire quelli che piangono e quelli che ridono.]

Espressione letteraria principale della cultura popolare di $\mathrm{Mi}$ lano e dei suoi dintorni fin dal Cinquecento, la 'bosinada' era una lunga poesia anonima monologata o dialogata in rima semplice e in dialetto che, dietro un tono generalmente comico, celava verità fondamentali della vita quotidiana, riflettendo la saggezza e il buon umore delle classi popolari lombarde. Tradizionalmente veniva cantata in piazza da un 'bosin,' contadino inurbanato. Pieno di proverbi e detti popolari, il suo contenuto era vario e poteva trattare qualsiasi argomento domestico che riguardasse il pubblico al quale la 'bosinada' veniva diretta.

I critici che l'hanno studiata sono piú o meno concordi nel vedere come tipici in questo componimento i seguenti caratteri: vernacolo, popolare, morale, realistico, linguistico e satirico; "composizione in versi gridata e recitata per la città dai cosiddetti 'bosin'," per citare il vocabolario di Cherubini. ${ }^{2}$ Sotto la voce 'bosin' poi, l'autore indica il contadino dell'Alto Milanese, spiegando inoltre 
che "cosí chiamansi fra noi quegli uomini che vanno per la città cantando e recitando quelle composizioni che son dette bosinad."3

L'unica generalizzazione sulla metrica che si possa fare è per la rima, che era quasi sempre $\mathrm{AABB}$. La forma variava parecchio, da quella "gridata e recitata" a quella stampata, nel formato di volantino, e generalmente recitata in piazza e poi distribuita o addirittura attaccata come grida ${ }^{4}$ ai muri. Quest'ultimo tipo era usatissimo nell'Otto e Novecento e la Biblioteca Braidense a Milano ne conserva ancora parecchi esemplari.

La fortuna critica della 'bosinada' non è stata sempre positiva. Gabriele Fantuzzi ad esempio la scarta, negandole una serietà lirica. ${ }^{5} \mathrm{Ne}$ dà un'opinione simile il Biondelli, nel concludere che la sola sua importanza "consiste nel documentare la storia patria, nonché lo spirito dei tempi."7

L'opinione di Fantuzzi, dato che non considera un possibile influsso storico di questa forma popolare, risulta alquanto semplicistica. Né sembra di poco rilievo il fatto che una forma di poesia, per quanto rozza di forma, sia riuscita a "documentare la storia patria, nonché lo spirito dei tempi," per dirla con Biondelli. Pare anzi evidente, senza volere entrare in argomenti sul valore letterario delle forme popolari in generale, che una forma spontanea e popolare, quantunque non sublimata, possa essere elevata da un letterato o avere addirittura esercitato un influsso sullo sviluppo letterario di un genere erudito; cosí Jacopone da Todi adattò la forma popolare della ballata, cosí la commedia dell'arte incise sul teatro comico erudito, e cosí anche la 'bosinada' può aver inciso sulla letteratura erudita dialettale di Milano. ${ }^{8}$ Sono convinto infatti che un legame stretto esista fra la 'bosinada' e la letteratura erudita dialettale e che tale legame si possa tracciare storicamente fin dal Cinquecento. Ritengo inoltre che l'evoluzione di questo legame, che nasce nel Cinquecento e che si realizza durante il Sette ed Ottocento nel connubio naturale dello spirito pratico e popolare della 'bosinada' e lo slancio sociale dell'Illuminismo, contribuisca in misura considerevole al carattere della letteratura milanese. L'intento di questo saggio è appunto di indicare questo contributo tramite uno studio del personaggio del 'bosin' corroborato da alcune considerazioni tematiche ricorrenti nella letteratura di Milano in dialetto.

Gli studi finora svolti sulla 'bosinada,' per essere stati accantonati dalla storia, sono scarsi e di interesse variabile. Uno dei principali 
è quello di Filippo Fichera ${ }^{9}$ che riporta un campionario delle 'bosinade' del tardo Settecento e dell'Ottocento esistenti nella Biblioteca Ambrosiana di Milano, dando ampia prova del modo in cui esse s'ispiravano alla storia contemporanea di allora. La raccolta è divisa secondo le forme monologica e dialogica. Nell'introduzione, Fichera vuole respingere la teoria che la 'bosinada' sia solo folclore, e perciò tiene a distinguere fra folclore/arte, cioè il prodotto della natura e dell'istinto delle persone e dei luoghi in cui vivono, e folclore/scienza, cioè quello rivelato e interpretato dallo studioso, per giungere a un rifiuto della posizione che il folclore non sarebbe arte. Le 'bosinade,' dice Fichera,

fanno parte del folclore milanese e lombardo, ma in quanto poesie, appartengono alla letteratura, e chi le studia è un letterato e non un folclorista. ${ }^{10}$

ed egli arriva finalmente a delinearne gli elementi cronachistici, condividendo l'osservazione del Biondelli, che essi sarebbero importanti quali specchio della società milanese:

Cronaca in versi, potremmo dirla. . . Carattere precipuo della 'bosinada' è la flagrante umanità del suo contenuto, cui aggiunge pregio l'immediatezza delle sue conclusioni. Vi si indicano luoghi, persone, e circostanze di comune conoscenza, aggettivi e appellativi e sentenze che suscitano la nostalgia. Essa è l'espressione del senso popolare, che osserva fatti del giorno e reagisce alle aberrazioni sociali. ${ }^{11}$

Come esempi dei fatti di cronaca che le 'bosinade' trattano, Fichera accenna al primo volo d'un pallone aerostatico a Milano nel 1784 che ispirò il senso poetico nell'autore di questo componimento:

El nost Orland el s'è alzaa come Astolf quan l'è andaa Coll'Ipogriff là nella luna per cercà a voeuna per una la bozzetta del cervell

Per guarí quel mattarell

Del grand Orland, che per amore

La voltaa 'l coo, l'è andaa in furor,

Come fan tanti person

Per infedeltaa di noter donn,

Ma per sicurass sempre de pu,

Iscambi d'Astolf el ghe andaa lu. ${ }^{12}$ 
[Il nostro Orlando s'è alzato

Come Astolfo quand'egli andò

Coll'Ipogriffo sulla luna

Per cercare

L'urna del cervello

Per guarire quel mattarello

Del grand'Orlando, che per amore

Gli diede di volta il cervello, andò in furore,

Come fanno in tanti

Per l'infedeltà

Delle nostre donne

Ma per esser sicuro di riuscirci

Andò Orlando invece di Astolfo.]

Ma generalmente le 'bosinade' mostrano uno slancio meno lirico e molto piú portato ad una descrizione concreta e immediata del fatto in considerazione. Sempre con l'esempio del pallone, l'ascesa della francese Mme. Blanchard il 15 agosto 1811, viene descritta in un'altra composizione di questo tipo che sottolinea la partecipazione entusiasta del popolo a questo avvenimento storico:

E con sto boccon de ver coragg

Mi gh'ho auguraa là el bon viagg

Com squasi tutt Milan

Ch'an daa segn ol batt i man!

[E con questo pezzo di vero coraggio

Le ho augurato il buon viaggio

Come quasi tutta Milano

Che ha mostrato la sua approvazione con battimani.]

Cosí come mette in risalto a mò di precisazioni geografiche di Milano l'immediatezza concreta del fatto:

L'han portaa anc mó nel Lazzarett

Che le sta là per on pezzet

Fintant che all'aquila han levaa

I ali, el coll senza fagh mal

Levand al ballon l'aria sta aciora

Lè tornada sanament

Coi pagn indoss bagnaa scorrent

Nell'ortaja sora ai proeus

Del local de S. Ambroeus. ${ }^{13}$ 
[Sono arrivati anche in Lazzaretto

Per un pezzo

Poi levando le ali

All'aquila e il collo,

Senza farsi danno

L'aria leva ancora il pallone

E torna sano e salvo

Con le sue tele fradice

Nell'orto e sulle aiuole

Di sant'Ambrosio.]

Ci sono tante altre 'bosinade' riportate da Fichera che descrivono fatti naturali, dalle eclissi ai terremoti, o che riflettono periodi specifici della storia milanese quali quello della Repubblica Cisalpina. La seguente, ad esempio, "Già che Pavia è liberada," elenca le stragi fatte dai francesi in quella città, e descrive, dall'ottica degli umili e con quel tono moraleggiante e con quella sentenziosità tipici di questa e di altre forme popolari, gli ovvi capovolgimenti dei principî filosofici della 'rivoluzione' portata dai francesi:

In quist $\mathrm{i}$ frutt avvelenaa

Dell'eguaglianza, e Libertaa?

In quist $\mathrm{i}$ patt, $\mathrm{i}$ vost reson,

$\mathrm{Da}$ salvà la roba e la Religion?

In quist $\mathrm{i}$ promess che $\mathrm{n}^{\prime}$ havii faa

De bona fed, de lealtaa,

De fratellanza, e de virtú

$\mathrm{D}^{\prime}$ aven liberaa de sciavitú

E tolt di sgrif di not tiran? ${ }^{14}$

[In questo il frutto avvelenato

Dell'uguaglianza, della libertà?

In questo i patti, le vostre ragioni

Per salvar la roba e la religione?

In questo le promesse che avete fatto

Di buona fede, di lealtà

Di fratellanza e di virtú

D'aver liberata dalla schiavitú

E tolto dalle sgrinfie del tiranno?]

L'ampiezza di temi che troviamo nelle altre 'bosinade' riportate da Fichera che abbracciano, insieme ai temi storici e politici, temi quotidianissimi come la cottura della polenta ed i rapporti fra moglie e marito, serva e padrona, stanno inoltre a sottolineare il ruolo di questo genere come vero simbolo della voce popolare. 
Dei tre studi sulla 'bosinada' che precedono quello di Fichera, il lavoro di De Castro ${ }^{15}$ copre tutta la letteratura popolare fino all'Ottocento e riporta alcune informazioni sulle poche 'bosinade' secentesche allora esistenti all'Ambrosiana ma sfortunatamente distrutte durante la Seconda Guerra Mondiale. Lo studio di Nalli ${ }^{16}$ fu invece un serio tentativo di catalogare le 'bosinade' archiviate nell'Ambrosiana e nella Biblioteca Braidense (e che avrebbe molto agevolato eventuali futuri lavori sul soggetto) ma purtroppo non piú valido per la distruzione e dispersione di molte delle 'bosinade' da lui analizzate. Infine l'articolo di Pizzagalli, ${ }^{17}$ come quello di Nalli scritto negli anni trenta, è importante per le informazioni sull'etimo e sull'origine della 'bosinada.'

L'origine di questo genere si riallaccia piú probabilmente ai cortei allegorici e ad alcuni componimenti teatralizzati del Cinquecento. ${ }^{18} \mathrm{I}$ primi seri tentativi di comporre in dialetto risalgono invece al 1560, anno in cui Gian Paolo Lomazzo fondò l'Accademia della Val di Blenio per incoraggiare il parlare in dialetto valligiano. Infatti, di quest'accademia si pubblicò Il Rabisch dra Accademiglia dor Compà Zavargna, ${ }^{19}$ raccolta di 'bosinade' e sonetti in dialetto scritti dai vari membri dell'accademia. Nessuno di questi componimenti risalta per particolare eccellenza lirica ma nell'insieme rappresentano un importante punto per stabilire sia la transizione della 'bosinada' dalla campagna alla città che il vero primo esempio di 'bosinade' stampate.

A differenza di quelle del Rabisch, le 'bosinade' che cominciarono ad essere stampate con regolarità nel Seicento rimasero quasi sempre anonime, tanto è vero che l'anonimità sarebbe infatti diventata caratteristica del componimento se la forma non fosse stata adottata da quasi tutti gli scrittori 'eruditi' in dialetto, a cominciare da Carlo Maria Maggi, famoso poeta e commediografo della fine Seicento. Nei secoli XVIII e XIX fiorí come genere e alcuni scrittori (ad. es. Fumagalli) godettero d'una discreta fama per le loro 'bosinade. ${ }^{20}$ Alcuni ricordano ancora d'averne sentite cantare ai primi di questo secolo, ma come genere scomparvero definitivamente durante il fascismo con i regolamenti della polizia urbana che vietava il tipo d'assemblamenti che generalmente accompagnavano la loro presentazione.

Fra i temi che troviamo piú spesso nelle 'bosinade ${ }^{21}$ predominavano gli argomenti domestici anche se, come si è visto sopra, il soggetto storico e politico non mancava, qualora i tempi lo permettessero. Vediamo alcuni temi preferiti in una poesia di Carlo 
Tanzi dedicata alla 'bosinada' ed impostata su un formato monologato:

Or nun faa quella sora al lott,

Sora ai tosann bon de nagott,

Sora di quij che fa lamor,

Sui poveritt che fa de sior,

Sora che i donn porta el scossa

Sora i moros mal pecenna

Sora el morneee, sora el sartí

Su certa razza de tenciia

Sora el mestee di litiga,

Su che i tosann spolpen i cà

Oh quanta gent ha stafilaa

L'invenzion di bosinaa! ${ }^{22}$

[Parliamo di quelle della lotteria

Sopra le ragazze buone a nulla, Sopra quelle che fanno all'amore,

Sopra le poverette che fanno le suore,

Sopra ciò che le donne portano

Sopra i morosi mal accoppiati

Sopra il mugnaio, sopra il sarto

Sopra certa razza di imbroglioni

Sopra il mestier di litigare,

Sulle ragazze che ti riducono in camicia

Oh quanta gente che questa

Invenzione della 'bosinada' ha fatto tribolare!]

Parole tematiche dello spirito popolare dunque - amore, il lotto, i battibecchi in famiglia, il sarto (cioè la moda) e il mugnaio (cioè il pane), gli imbroglioni. A questa lista parziale offerta da Tanzi possiamo aggiungere le feste pubbliche (e i problemi d'ordine civile che ne conseguivano) ${ }^{23}$ frammisti v'erano pure i temi della campagna, dove la 'bosinada' aveva la sua origine - molte parlano della vendemmia e ce n'è una perfino sulle patate. Con l'imborghesirsi del genere, diventa comune cantare le lodi delle osterie milanesi. Tutti temi, insomma, della cronaca quotidiana, alcuni piú interessanti di altri ma che insieme intrecciano un panorama della vita popolare che, da quella rustica brianzola diventa, man mano che il 'bosin' s'addentra nei quartieri poveri della città e li fa suoi, cittadina e spiccatamente milanese in tonalità. Grazie a questo contatto urbano, la 'bosinada' prende spesso uno slancio civico, tendenza che riflette le caratteristiche d'immediatezza e di 
realismo alle quali molti hanno accennato come tipiche di tutta la letteratura lombarda.

\section{IL BOSIN}

La prima tappa d'obbligo nel tracciare il rapporto tra questo genere e la letteratura erudita in dialetto consiste nel vedere l'evoluzione del suo portavoce da cantastorie a quella maschera milanese tradizionalmente attribuita a Carlo Maria Maggi, 'Meneghino,' ed oltre. Data la scarsezza di 'bosinade' secentesche sopravvissute ai bombardamenti della Seconda Guerra Mondiale, la posizione storica di Maggi alla fine di quel secolo assume infatti un'importanza chiave per lo studio del rapporto tra 'bosinada' e letteratura dialettale erudita, come vedremo.

Bertoldo, Barba Togn, Baltram, Meneghino - ecco i nomi piú comuni usati dai cantastorie quando le dicevano, e/o dai compositori, anonimi o non, quando scrivevano le 'bosinade.' Ognuno di questi nomi godette all'interno del genere d'un periodo di voga, il che ci agevola il tracciare l'evolversi della figura come portavoce. La maschera milanese, ad esempio, incomincia a formarsi già nei tempi dei longobardi nel personaggio rustico di Bertoldo, nome che adoperavano i cantastorie, facendone gradualmente un tipo che poco a poco si divulgò nelle città e nelle campagne lombarde. Un servo in possesso di quella grossolana saggezza e in cui il volgo s'identificò, Bertoldo divenne in Lombardia, cosí come in Germania, la personificazione dell'astuzia contadinesca degli altri 'bosin' di cui è certamente progenitore. ${ }^{25}$ Bertoldo stabilisce la stretta relazione fra sé e la miseria della classe popolare lombarda costretta a combattere con umore, lazzi e sarcasmo, gli oltraggi dello straniero e la povertà degli oppressi; una relazione fra l'altro attestata da tanti letterati che, in varie epoche, hanno sfruttato questo personaggio germanico. ${ }^{26}$

Nel tardo Cinquecento il 'bosin,' che continua la tradizione stabilita dal cantastorie Bertoldo, diventa Compà Zavargna, personaggio inventato da Gianpaolo Lomazzo nel suo Rabisch soprammenzionato. Anche Zavargna è contadino, e dimostra alcune di quelle caratteristiche che abbiamo visto in Bertoldo e che vedremo espresse in altri 'bosin' a venire. ${ }^{27}$

Te se compà Zavargna che tra vugn

Pum diviso senza simulaciogn

Che varen piu de tí no gh'è nisugn. ${ }^{28}$

[Te sei quel compare Zavargna che 
Va giú piatto

Non c'è nessuno che valga piú di te.]

Questo aperto parlar ingenuo cela talvolta una satira pungente come quella che troviamo nella lunga 'bosinada' "Remò scia fà de la prematica in Miran," cronaca ampia di diversi aspetti della vita milanese e insieme critica umoristica delle gride. Già dal titolo si vede Zavargna — già Bertoldo - a Milano fra un popolo milanese ormai disposto, dopo secoli di poesie in dialetto di scrittori come Pietro da Bascapè e Buonvicino da Riva (Bonvesin della Riva), ad accogliere con entusiasmo le sue sentenze. Che il 'bosin' si fosse solidamente stabilito come personaggio rappresentante lo spirito popolare in tutta la Lombardia, ce lo afferma il caso d'un individuo impiccato ad Alessandria nel 1628 per aver recitato una 'bosinada' critica degli spagnoli. ${ }^{29}$ A Milano Zavargna diventa il 'bosin' Baltram de la Gippa o da Gaggian, che veniva portato in piazza ormai agli scorci del Cinquecento da attori professionisti come Nicolò Barbieri che, insieme alla moglie che faceva Baltramina, moglie di Baltram, presentava 'bosinade' dialogate. Questo Baltram sarebbe diventato allora quasi un nome collettivo per 'bosin,' una specie di maschera del tipo stesso, nativo oramai di Gaggiano sulla riva destra del naviglio grande:

L'indole di Baltram è tale quale i tempi lo fecero. È un contadino goffo, impacciato, ignorante, eppur sentenzioso; non sa che lamentarsi e piangere; non va mai in collera; rispettoso verso i governanti e tutto spera da essi. ${ }^{30}$

Durante questo periodo, alla fine del Cinquecento, si ha un primo esempio di come alcuni poeti eruditi di Milano avrebbero cominciato ad identificarsi col personaggio del 'bosin.' Fabio Varese, compositore musico del Duomo, scrisse allora poesie in dialetto che rimasero inedite fino al lavoro di Cherubini del 1816. In un sonetto Varese canta ironicamente la propria povertà, presentando se stesso, anche in questa forma metrica elevata, come tipico 'bosin' delle strade di Milano:

Me rallegher con ti Fabi Vares,

Ghitarra sbotasciaa, cavagna rotta,

Che per drovà la lengua adree a ona $\mathrm{p}_{3 \mathrm{i}}$... .

No gh'è on par tó su tutt el Milanese.

[Mi rallegro con te Fabio Varese 
Chitarra spaccata, scatole rotte

Ché a parlare con le puttane

Non c'è par tuo in tutto il Milanese.]

Da Baltram e dai 'bosini' cittadini come Fabio Varese si salta facilmente a Meneghino, il personaggio di Maggi chiaramente radicato nella tradizione della 'bosinada.' Servo d'origine cittadina, non piú tanto goffo, anzi piú furbo nel sapersi barcamenare nel mondo del padrone, essendosi imborghesito come personaggio, ha ormai alle spalle l'esperienza della città; impacciato ed ignorante talvolta in superficie, si mostra ciononostante sentenzioso e moraleggiante contro i malcostumi della Milano spagnoleggiante del tardo Seicento, ${ }^{33}$ anche se la sua satira rimane in larga misura bonaria. Come si sa Maggi, con questo personaggio, diede una grande spinta al teatro milanese, ma come si vede in una sua poesia, "Il bosin orb," che precede le commedie in cui creò Meneghino, s'ispirava già al 'bosin' come portavoce del suo pensiero:

L'olter dí, ch'era stà per tut Milan,

Vedend uga, zucchet e peveron,

Tornava a cà sul bass inscí pianpian,

Dondand con on vist, che ona canzon;

$\mathrm{E}$ anca mi curios mettè giò i scorb,

Per senti la canzon de Bosin orb.

[L'altro giorno che gironzolavo per Milano,

Comprando uva, zucchine e peperoni,

Tornavo stanco a casa piano

C'era gente che stava a veder uno che cantava

E essendo anch'io curioso misi giú la scorta,

Per sentir la canzone del bosino cieco.]

Ciò che segue nella poesia riflette la stessa filosofia fatalista del 'bosin' com'era fin da Bertoldo, la filosofia della commedia Il manco male e di tutte le commedie del Maggi. Là l'io narratore diventa Meneghino. Qui, l'autore ha già un'ovvia simpatia per il 'bosin' che la gente ascolta. Le somiglianze fra questa poesia e il 'manco malismo' delle commedie che verranno piú tardi sono talmente evidenti che ne riporto un brano centrale abbinato ad alcune battute di Cricca e Meneghino della ballata a conclusione del manco male perché il lettore possa vedere la continuità d'ispirazione fra le due: 


\section{1. "Il bosin orb"}

De spess a cercà tant, e cerca rogna E vedem, per sta mej, che se pegiora Fà per il ciel, sem pur i gran maszoc, A cercà in tera el paradis de oc! ${ }^{34}$

[Spesso, dal cercare tanto, si cerca anche rogna

$\mathrm{E}$ vediamo che, per stare meglio, restiamo peggio invece Cercando d'arrivare troppo in alto, siamo scemi, A cercare di veder in terra il paradiso!]

2. Ballata per Il manco male

I cavaij, che corren trop

Duren poch e dan al fianch, Lassè andà cosí a pè zopp,

Che la va né pú né manch. ${ }^{35}$

II cavalli che corron troppo

Duran poco e si sfiancano,

Lasciateli andare cosí a pie' zoppo

Che la va né piú né manco.]

Il fatalismo nascosto dietro l'umorismo di questi versi diverrà piú accentuato nel personaggio di Meneghino man mano che egli diventa il personaggio centrale delle commedie di Maggi: la sentenziosità, la furberia, l'ingenuità, spiccano pure in altri personaggi, specialmente in quello di Baltramina, il cui nome suggerisce il legame con Baltram de la Gippa sua moglie e che simboleggia il buon senso popolare. Baltramina recita i prologhi e gli intermezzi delle commedie I consigli di Meneghino e Il falso filosofo, fuori e al di sopra dell'azione di queste pièces, e nel commentarne la morale secondo la tradizione del 'bosin' diventa una specie di musa della letteratura del Maggi e dello spirito popolare che l'autore vuole esaltare. Nella commedia dialogata Il concorso de' Meneghini è Baltramina stessa a stabilire il compito del 'bosin' teatrale 'Meneghino,' dandoci con ciò un esempio dello stretto legame fra la letteratura erudita dialettale e quella popolare di Bertoldo Zavargna e Baltram che abbiamo esaminato fino al Maggi. Le parole di Baltramina a proposito del dialetto e del 'bosin' che lo canta sono significative:

Ma 'l só don prinzipael

L'è la fazilitàe de fà capí

Cont esempij, panznegh, e proverbij

I pú sublimm conzett 
De' gran Filosofon finna in Brovett.

Studiè i mè Toson:

Desí robba, che edifica, e che giova

Anch in del menà baij,

Che allora la's rezev con pú larghezza

In del slargass el coeur par l'allegrezza.

Diga chi voeur, l'è questa

L'art vera del parlà;

L'eloquenza da i coss,

E no da i sciansc la ven:

Desí del bon, che dirí semper ben.

E che onor sarà 'l vost, se i forestè

Vegnaran a pescà

In d'i Meneghinaed i bej pensè. (Consigli, vv. 906-23)

[Ma suo dono principale

E la facilità con cui riesce a far capire

Con esempi, favole e proverbi

E concetti piú sublimi

Dei grandi filosofi anche in Broletto.

Studiate ragazzi miei:

Dite.cose edificanti ed utili

Anche quando prendete in giro,

Ché siamo piú aperti a riceverle

Quando il cuor s'allarga nell'allegrezza.

Si pensi ciò che si vuole, è questa la vera arte di parlare;

L'eloquenza è nelle cose

Non nelle ciance:

Dite del buono che direte sempre bene.

E che onore sarà il vostro se i forestieri

Verranno a pescare

Buoni pensieri nelle nostre 'meneghinate.']

Meneghino cioè, usando la lingua del popolo e i temi con cui esso è familiare, deve portargli dei concetti utili ed edificanti, come facevano i 'bosin' prima di lui. La frase "l'eloquenza da i coss/E no da i sciansc la ven" sta a ribattere sul carattere concreto ed immediato del concetto, elemento essenziale pure alla natura della 'bosinada,' come abbiamo visto. Se ciò non bastasse, il termine 'Meneghinaed,' le sentenze d'una figura popolare cioè, può chiaramente essere visto come sinonimo di 'bosinade,' e mi pare mostri senza dubbio una continuità che lo scrittore vedeva fra il suo personaggio e quello del 'bosin.' Ricordiamo a proposito i commenti di De Castro sulla sentenziosità di Bertoldo contro gli stranieri dei secoli precedenti; qui le parole "Bej pensè" che "i forestè" possono trovare nelle 'meneghinate," suggeriscono infatti che Maggi, durante il dominio spagnolo in cui egli scrisse, volesse 
proprio stabilire un nesso fra Meneghino e quel portavoce originale dell'umile messaggio delle 'bosinade.'

Questo legame col 'bosin,' evidente nel Maggi, diventerà caratteristico anche nei susseguenti poeti dialettali piú rinomati di Milano. Soffermiamoci brevemente per vedere, tramite pochi esempi degli scritti di questi, come si riconoscono debitori a Maggi per il retaggio di morale e spirito popolari insiti nelle 'bosinade' che egli era riuscito a tramandare nel suo Meneghino:

1. Girolamo Birago ${ }^{36}$ fa recitare il prologo della sua commedia Donna Perla da Meneghino che allude in esso ad alcuni passi de Il falso filosofo e de $I l$ barone di Birbanza del Maggi. ${ }^{37}$

2. Pietro Cesare Larghi, ${ }^{38}$ primo poeta dialettale di rilevanza dopo il Maggi, chiamando se stesso 'meneghin' nel suo sonetto "al Sur Cardinal Arcivescovo Pozzbonell," ${ }^{39}$ ci dà un esempio del costume dei poeti dialettali di identificarsi nella tradizione del personaggio ("Adess gibilleam tucc, ma pu de tucc, / El vost serv Meneghini pie d'allegrezza" [Adesso giubiliamo tutti, ma piú di tutti / Il vostro servo Meneghino pieno d'allegrezza]).

3. Stafano Simonetta, ${ }^{40}$ in un altro sonetto alla morte del gatto del suo amico Domenico Balestrieri, si riferisce a questo amichevolmente con il nome di Meneghino ("O che brutta disgrazia, Meneghin; / Savii coss è success al vost gattin." ${ }^{11}[\mathrm{Oh}$, che brutta disgrazia, Meneghino, / Sapere ciò che è successo al vostro gattino]).

In questa poesia 'Meneghin' e 'bosin' sono entrambi usati come sinonimi di poeta, prova ulteriore del filone di continuità del quale si parla. È un filone peraltro che non si dilegua attraverso il secolo come possiamo costatare negli scritti di due dei piú conosciuti poeti dialettali milanesi del secolo, Carlo Tanzi ${ }^{42}$ e Domenico Balestrieri. ${ }^{43}$

4. Carlo Tanzi, ad esempio, nel suo "Recitaa in l'Accademia sora l'Invenzione di Bosinada" 44 attribuisce il merito della poesia erudita in dialetto al 'bosin':

Se no ghe fuss staa al mond Bosin,

S'sciavo sur rimm de Meneghin,

Dov vorevem toeunn l'ideja?

Fassela dà de l'Omm de Preja? (Cherubini, Collezione V, p. 52)

[Se il 'bosin' non fosse stato al mondo,

Dedicato a fare le rime in 'meneghino,'

Da dove avremmo tolto l'idea?

Forse da una statua?] 
e traccia l'evoluzione della 'bosinada' accennando a tutti i poeti che abbiamo menzionato qui, dai 'bosin' del Cinquecento fino ai nostri giorni.

5. Domenico Balestrieri, coetaneo del Tanzi e il piú famoso dei poeti dialettali del secolo, indica Baltram come modello: "scusaroo / Col fà la mia comparsa de Baltram" [Mi scuserò / Facendo la mia comparsa da Baltram], e afferma la sua intenzione di continuare a scrivere, da 'bosin,' in 'meneghino': "Giacché hoo el nom / faroo i vers de Meneghin [Giacché ho il nome, farò i versi in 'meneghino' $],{ }^{45}$ riconoscendo infine Maggi come vate della poesia erudita dialettale

El Magg, che in tutt el rest l'è el to maister

L'è andaa lu inan, l'ha faa lu la calada Sul bon sentè. . . ${ }^{46}$

[Il Maggi, che in tutto il resto è il tuo maestro

È stato lui a mostrare la via, a fare scalo

Sul buon sentiero.]

Inoltre, in una bosinada intitolata "La Badia de Meneghitt," Balestrieri attribuisce al Maggi il merito d'aver assicurato la sopravvivenza del genere stesso, riportando parecchie frasi dell'autore che segna con asterischi. ${ }^{47}$

La "Badia" di Balestrieri ricorda molto Il corcorso de' Meneghini su cui è indubbiamente modellata, e per il modo in cui cerca di gloriare l'uso del dialetto e perché tutte le frasi maggesche che egli usa sono levate da quell'opera. Balestrieri calca sull'eredità e sulla continuità letterarie tramandate da poeta in poeta prima e dopo Maggi, cominciando dal letterato Giovanni Capis, autore, nel 1606 , della prima opera di prosa in dialetto, ${ }^{48}$ Varon de Milan $^{49} \mathrm{e}$ proseguendo con una rassegna delle figure piú eminenti nel campo dialettale, come aveva fatto Tanzi nella sua poesia sull'invenzione della 'bosinada':

Ma in di bej sottigliezz del nost Varon,

In di vers milanes,

Che ha scritt antigament Fabi Vares

In quij del fint Pedrin,

Del Largh, del Simonetta,

E for el tutt del Maeg, quel gran Poetta 
No'l ha dir del Toscan, né del Latin,

Tugg i noster paroll, s'hin mis a loeugh

Per spiegà i bej penser, hin semper bej.

$\mathrm{E}$ che onor farà el nost se $\mathrm{i}$ forester*

Vegnaran a pescàa*

In di Meneghinaed, $\mathrm{i}$ bej penser*

Come l'è già suzzess, e 'l pomm provà. ${ }^{50}$

[Ma nelle belle sottigliezze del nostro Varon,

Nei versi in milanese

Che ha scritto tempo fa Fabio Varese

In quelli del finto Pedrin

Del Largo, del Simonetta

E soprattutto del Maggi, quel gran Poeta

Che non ha usato né il toscano, né il latino,

Bensí le nostre parole in dialetto

Per spiegare dei pensieri sempre belli.

E che onor sarà il nostro se i forestieri*

Verranno a pescare*

Nelle nostre 'meneghinate' i bei pensieri*

Com'è già successo, e continueremo a vedere.]

Pare perciò evidente da quanto sopra che alcuni dei principali poeti dialettali del Settecento riconobbero una vera tradizione poetica popolare fiorita nel Cinquecento col 'bosin,' solidificata e tramandata fedelmente dal Maggi attraverso il suo personaggio Meneghino.

\section{LA TEMATICA}

Il raporto fra 'bosinada' e poesia dialettale erudita che siamo venuti tracciando nel personaggio del 'bosin' si può anche confermare prendendo in considerazione l'aspetto tematico.

I primi scritti che piú rassomigliano in forma e spirito alla 'bosinada' sono quelli di Gianpaolo Lomazzo, ${ }^{51}$ che è anche il primo poeta a scrivere in dialetto milanese, segnando cosí l'imborghesirsi del dialetto e degli inizi della letteratura dialettale di cui sopra s'è già brevemente parlato. Nella sua lunga poesia soprammenzionata "Remò scia fà de la prematica in Miran" l'autore, prendendo come spunto una delle famigerate gride del Cinque e Seicento che cercavano a mò di pesanti tasse (con i risultati di cui la storia è testimone) di ridurre le industrie di lusso e regolare anche la moda, arriva a parlare, sempre con quel lungo raccontare cosí tipico della 'bosinada,' di molti temi che riappariranno negli esemplari sette o ottocenteschi che si possono leggere ancora. La 
poesia in sé è un commento interessante sulle gride in genere e in particolare sulla moda (uno dei molti temi di questa poesia) che sarà in seguito uno dei soggetti preferiti dai poeti eruditi. In essa Lomazzo inizia compatendo le vittime della grida sulla moda con ironia, e discute con ricchezza di dettagli dell'industria e di altri oggetti di lusso tipici fra le classi ricche di quei tempi, offrendoci non solo un commento satirico ma anche una vera cronaca delle mode d'allora.

Poi però, dopo una lunga descrizione delle frivole mode del tempo, l'autore assume un tono serio, parlando anche delle osterie, punti di ritrovo del 'piccolo popolo.' Quelle, diventando piú care, toccano piú da vicino la gente delle classi basse. Con il tono moraleggiante della 'bosinada' Lomazzo si lamenta dei prezzi alti del mangiare in genere e del grave effetto che questo fatto ha sulla gente che ne soffre di piú:

Ma parlemmm mo sora ai ost

Dove ghe va tanta gent

A mangià, che verament

Fan sta maa la soa bregaa,

Anca lor ha d'ess manàa

Con quij oltr' in ordenanza

E tucc quij che stà sul Broeu

Quij che stan poèu press al Domm,

Che voeun fà coi recatton,

Che no s'pò mangià pú bon

Nanch on frut, a poèu lu tant car:

Faran ben a fagh reparar.

[Ma parliamo un po' sulle osterie

Dove va tanta gente

A mangiare, ché veramente

Fanno stare male la brigata,

Anche loro devono vedere di

Imporre le ordinanze

Tutte quelle di Broletto

E quelle vicino al Duomo

Ché vogliono fare anche coi fruttivendoli 
E non si può piú mangiare bene

Neanche la frutta, ch'è ora tanto cara:

Dovrebbero porvi rimedio.]

Dall'immediatezza stabilita dai riferimenti geografici al Broletto e al Duomo, l'autore passa a suggerire che le autorità farebbero bene a regolare anche i venditori di quella merce i cui prezzi incidono di piú sui poveri, e si lamenta in continuazione di quelle misure proposte che colpiranno piú pesantemente i poveri, come quella che vieta che il pane superi una certa misura di grandezza: "Per el prim voeuren mett fren / Ch'l pan sia on poo pu gross." [Come prima cosa vogliono imporre / Il limite sulla grandezza del pane] suggerendo persino una reazione da parte del popolo a questa misura:

L'ha 'ess una maraveja

Se non nass on badaluch

Sul cercâ nobiltà

Dov'la ved tant mesturaa

Che mi tasí a voo da qui. ${ }^{52}$

[E una meraviglia

Se non ne nascon chiassi

Sul cercare di giustificare

Tanti sbagli

Che non ne voglio parlare.]

Oltre alla netta presa di posizione a favore del popolo a cui potevano importare poco le misure prese contro il lusso, ci colpisce la tendenza dello scrittore a descrivere con concretezza dovunque nei versi di questa poesia la vita quotidiana, che viene delineata sotto molti aspetti - dal carovita e dalla severità con cui essa incide sul popolo, alla prostituzione e alla confusione del sistema criminale, che pure sono discusse in dettaglio. Il tono della poesia infine, specie quando insiste nel dare piú considerazione al popolo, è quello moraleggiante che vedremo nelle 'bosinade' del Sette e dell'Ottocento.

Le uniche prove che abbiamo della continuità tematica della 'bosinada' nel Seicento ci vengono attraverso brevi commenti che ne fa De Castro. Gli esemplari che si trovavano all'Ambrosiana facevano parte della Raccolta di bosinate ed altre poesie in dialetto milanese, distrutta e/o dispersa durante la Seconda Guerra Mondiale. De Castro ne descrive alcune e ne commenta brevemente il conte- 
nuto in modo da darci un'indicazione di come la 'bosinada,' quale genere, continuò ad essere popolare fra la gente per tutto il secolo. Riporto la lista di De Castro per intero:

1. Nova Cipollata in lingua rustica milanese (1616).

2. Allegrezza fatta da Baltram da Gaggiano sopra la bondanza in lingua rustica milanese (s.d.).

3. Il lamento del contadino sopra diverse arti (s.d.).

4. "Navareida" descorso intorno a la resa de Breda in despresij di novarin nostran, dà in lus da Battista de Miran quest'ann 1625.

5. Lamentazione che fanno Beltram da Gasina da Gorgonzola e Bausion da Gorgonzola sopra li presenti tempi calamatosi (1630).

6. "Bradaneida" ragionamento fatto in lode di Bradà di Porta Nova dove si tengono tutti i bredà, feste giochi e fuochi fatti in Milano per l'allegrezza della presa di detta Bredà (s.d.).

7. La nova bosinada sora $i$ spas del carnevaa nuovamente composu d'un bosin non cognossu che se vur fa mott in vos per es dod'a da tugg $i$ spos (s.d.). ${ }^{53}$

I titoli ci offrono alcune indicazioni degli sviluppi storici discussi sopra: le prime due indicano l'uso del milanese rustico nelle 'bosinade' ancora ai primi del secolo; la terza il contadino alle prese con la città; la quarta invece ci interessa sia per il titolo, che indica che il bosin s'è stabilito ormai nella città di Milano, sia per il tema politicizzante, significativo in quanto anti-francese ma non antispagnolo. La critica anti-spagnola era infatti praticamente impossibile, come abbiamo visto dall'episodio del 'bosin' ad Alessandria. Comunque, il titolo di questa 'bosinada' segna senz'altro l'inurbanamento definitivo del 'bosin' a Milano. La quinta ci suggerisce, coi "tempi calamitosi" del suo titolo, una probabile descrizione della peste di quell'anno. Invece, De Castro si limita ad accennare solo a quello ch'egli ritiene l'elemento anti-spagnolo del componimento - una protesta, secondo lui, ma "un lamento a bassa voce, rispettoso, ${ }^{154}$ in cui non viene accusato nessuno, in cui l'accusa la fanno i soggetti solo attraverso il loro lungo raccontare dei fatti. Ne riporta alcune strofe indicative:

Chi saves i dané spess

Senza quel che sta robà

A le con cas degn de pietà

Di dané ch'han portaa via.

[Chissà poi i soldi spesi

Senza parlare di quel ch'è stato rubato

Ed è un caso di pietà

I soldi che hanno portato via.] 
dove l'obbrobrioso sfruttamento da parte dell'oppressore della città occupata si sente forte anche in pochi versi per l'insistere di chi scrive sulle quantità di beni e denari pignorati e portati via.

Gli ultimi due componimenti menzionati da De Castro trattano del carnevale e di simili feste, che fungono spesso da sfondo o da tema nelle 'bosinade' e sottolineano il sovrammenzionato imborghesirsi del genere.

Il culmine dell'evolversi degli elementi a cui questi titoli accennano sarà nell'opera del Maggi. Abbiamo già visto come egli s'ispiri al 'bosin' in una poesia, per poi creare nel suo teatro il proprio 'bosin' Meneghino. Maggi imposta le sue opere teatrali su una base cronachistica e dà loro sempre un tono moraleggiante, con speciale attenzione alle cose quotidiane, dandoci cosí uno specchio assai vivo dell'ambiente domestico e di quello della città di Milano. Ne Il concorso de' Meneghini la musa Baltramina ammonisce i candidati promossi di portare in Broletto con esempi, favole e proverbi i concetti utili ed edificanti, indicando come strumento dell'insegnamento il dialetto popolare. Pervade tutti i discorsi di Baltramina al riguardo quel tono morale tipico della 'bosinada' che spinge il Meneghino/'bosin' a portare il suo messaggio al popolo. Come vedremo piú oltre, questo stesso atteggiamento sarà poi quello adottato dai poeti dialettali settecenteschi, stando al De Castro, ${ }^{56}$ ma è anche tradizionale della 'bosinada' del Cinquecento come abbiamo visto nel Lomazzo, e di quella del Seicento. È, comunque, il modo preferito anche dal Maggi, il quale imita lo stile e la tonalità della 'bosinada' facendo largo uso, nei prologhi e negli intermezzi, d'un personaggio come Baltramina per tirare le somme morali della pièce fino a quel punto ${ }^{57}$ oppure per parlare d'un tema 'ridicoloso's8 come quelli di tante 'bosinade.'

Ma nel suo 'raccontare' il Maggi rivela, cosí come si era soliti vedere nelle 'bosinade,' una sorta di impegno, anche sotto una patina talvolta frivola e ridicola. Prendiamo come esempio un intermezzo raccontato da Meneghino che allude agli spagnoli:

Quist, che governa i olter,

Par quel, che me cutaeva on gentiromm,

Se vorressen d'ul ver d'i soéu magon,

Fan invidia, e faraevan compassion.

Prummma quel, c'ha i command,

$\mathrm{Al}$ s'ha da tengí lu nett comè on spegg.

El spegg l'ha quest, c'anch in so dagn l'è chiaer,

E che ogni poch pelucch a ghe compaer. 
L'ha da guardass d'ogni leggier pecchàe,

Che se ghe 'n scappa vun,

Subet al se fa vizij del comun.

(Intermezzo dell'ambizione, vv. 88-99)

QQuelli che hanno il governo degli altri

Mi raccontava un gentiluomo

Se volessero dire il vero dei propri magoni,

Fanno invidia e farebbero compassione.

Innanzitutto chi ha il comando,

Deve serbarsi pulito come uno specchio.

Lo specchio ha questo di particolare, ch'è trasparente anche a proprio [danno,

E che ogni minima magagna si vede.

Deve guardarsi da ogni anche piccolo peccato

Ché se gliene scappa uno,

Il vizio è subito sulla bocca di tutti.]

Come satira non è male, data la necessità ovvia dell'autocensura. Si tratta di ammonimenti miti che ricordano che il popolo osserva tutto quel che fa il governo, e di una presa in giro delle leggendarie gride beffate come ai tempi di Lomazzo:

Se suzzed on travers,

Che sempre en suzzed,

Subet solten su tregg: no se prove?

Vun vorraev fà ona cria,

Con mett on' impennàe d'i presj che sia.

(Ambizione, vv. 104-07)

[Se succede qualcosa di storto,

Come ne succede sempre,

Saltan tutti subito sú: Perché non ci si pone rimedio?

Magari ci fosse una grida,

Che ci mettesse le pene piú severe.]

Altri notevoli esempi di questo lungo 'raccontare rispettoso' si trovano nelle descrizioni delle condizioni della prigione a Milano e della confusione che regnava nei tribunali (il falso filosofo, II, 13; II, 10). In altra occasione Maggi cela una satira azzeccata contro le condizioni economiche dei poveri in un discorso che fa al suo ricco padrone borghese (I consigli di Meneghino, I, 2). Si potrebbero citare tanti altri esempi di questo lungo 'raccontare' in cui si fondono satira mite e cronaca quotidiana. Piuttosto, per raccogliere i fili della continuità del rapporto fra 'bosinada' e letteratura dialet- 
tale erudita anche nella tematica, vediamo brevemente come perdura il legame anche dopo Maggi.

Basterebbe guardare un elenco delle novellette del Balestrieri per convincersi che quell'ingenuità di Bertoldo, quel cauto buon senso di Baltram, quel "manco malismo" e moralismo di Meneghino erano sopravvissuti fino al Settecento ed oltre: "Valutà i coss per quel che varen" [Valuta le cose per quel che valgono], "Senza ess cercaa no se da consej" [Non si danno i consigli se essi non sono sollecitati], "In bocca ciusa no gh'entra mosch" [In bocca chiusa non entran mosche], "L'ingoddisia no l'è mai contenta" [La ghiottoneria non è mai sazia], "Mesurà i coss con l'istess brazz" [Bisogna misurare le cose con le proprie braccia]. In questi pochi versi già vediamo un forte spirito pratico e di buon senso.

Ma teniamoci in modo specifico alla tematica, per vedere come i poeti dialettali eruditi fossero ligi alle 'bosinade' anche nella loro scelta dei temi preferiti. Per forza di cose bisognerà qui limitarci a una scelta piuttosto ristretta di poesie e scrittori. Iniziamo allora con Maggi, Tanzi e Balestrieri per arrivare fino a Porta. Le 'bosinade' che ho scelto sono generalmente senza data, anche se tutte, a giudicare da alcuni accenni storici, geografici, ecc., debbono datare agli ultimi anni del Sette a ai primi dell'Ottocento. Sono tutte esistenti nelle raccolte della Biblioteca Ambrosiana o nella Braidense, a Milano.

La Moda. La moda viene beffata per i suoi eccessi nelle 'bosinade' fin dal Cinquecento. Un buon esemplare a questo riguardo è "La noeva bosinaa sulla moda malandrina di vestí alla guigliotina," ${ }^{59}$ che, per descrivere una delle esagerate acconciature femminili del tempo, usa una ricchezza di dettagli che ricorda la lunga descrizione della moda nella "prematica" del Lomazzo. La 'bosinada' che cito qui è tipica nel suo tono satirico:

Ora i veder col frontalett

Com'è i angeritt di Cattallet.

Forma a rebesce con simetria

Lavorga d'or del sciur mattia;

Con duu cennelon d'or nett e s'cett

Per grand de' quii ch'è la al Brovett. ${ }^{60}$

[Ora i vetri col frontale

Come i puttelli di Cattallet.

Fanno a rebesco con simmetria

Opere d'oro per la signora mattarella;

Con due reti d'oro netto e schietto

Fra le piú grandi del Broletto.] 
Tanzi, in un componimento di terzine "Sora la spelorciaria," redarguisce la gente ad osservare la moderatezza nel vestire e critica il tipo di eccesso che questa 'bosinada' descrive:

Em de vestiss? Per mi no foo desvari

Del velu al baracan: basta quaciass

I carna, e basta reparass de l'ari.

Ero besogna trà el vestii in sconquass

In manch de quella come cert mincion:

O gram o bon ch'el sia, andemm de pass. ${ }^{61}$

[Quanto al vestire? Per me non faccio disvari

Dal velluto alla lana: basta coprirsi

Il corpo, ripararsi dalle correnti d'aria.

Non c'è bisogno di mettersi in ghingheri

Per far colpo come fanno certuni:

Comunque ci si vesta, si va a spasso.]

Servo/Padrone. Il rapporto servo/padrone è un tema che appare spesso nelle 'bosinade' dialogate; la forma della botta e risposta, per via dell'immediatezza del rapporto fra i dialoganti, presenta in modo ideale la situazione dei poveri alla mercé dei capricci dei datori di lavoro. Nella sua commedia Manco Male, Maggi ci dà un'idea della situazione da servo del suo famoso 'bosin' Meneghino:

Par trè liver al mes

Con trí popoèu merdos,

E on mezz de quel da trí da collazion,

Che no meritten gnanch

Da mett sgio 'l farioèu

La voeur che 'gh tegna nett fin l'aquairoèu.

(il manco male, III, 11)

[Per tre lire al mese

Con tre merdosi crostini

E un mezzo di quello da tre soldi per colazione,

Che non meritano neppure

Di dover metter giú il ferraiolo

Vuole che le tenga pulito anche il cesso.]

Nella 'bosinada' che segue, scritta nel 1812, la padrona fa licenziare il servo dalla cameriera. Questa azione compiuta per procura sottolinea il potere di chi comanda e la miseria di chi è comandato. Le diverse fatiche descritte dal povero servo sono testimo- 
nianza d'una vita squallida poco cambiata dai tempi di Maggi, anche se la paga è leggermente aumentata da allora:

CAMERIERA: Ma cosa serva tant reson;

No i faa che la vostra obbligazione

Se ten per quest el servitor

Per fa el besogn tutto quant occor.

SERVO: $\quad$ E per ciappà poc o nente

La da fa'l coeugh e anca el squatter

Andà col gerla al gran Tajater?

Fam sgurà fina i quadrii,

Fa de croteur di sciavvatin

De sart, de servo e de facchin,

Vojà, nettà vas a boccaa,

Fa tant lett come all'ospedaa,

Fa 'l pancott e scovà i sal

E mett perfina i servizial

Con tant da fa, con tanti pes

Per ciappà sett lira al mes,

Giacché a serví sta sort de piagh

No gh'è sui spes d'andà sul lagh. ${ }^{62}$

[C: Ma perché tante storie?

Voi non fate che il vostro obbligo

Il servo c'è per quello

Per far tutto quel che occorre.

S:

E per intascare poco o niente

Deve fare da cuoco e da sguattero

Portare la scorta con gli spuntini al teatro?

Pulire perfino i quadri.

Fare il bucato

Fare il lavoro da ciabattino

Da sarto, da servo, da facchino

Svuotare, nettare la padella

Fare tanti letti come ne ha un ospedale,

Fare il pane, scovare il sale

Amministrare perfino il clistere

Con tanto da fare, con tanti pesi

Per pigliare sette lire al mese

Giacché a servire questa sorta di disgraziata

Non vi si guadagna neanche per andare sul lago.]

Il lotto. In una specie di intermezzo lungo, Il lotto di Genova, Maggi ci aveva dato un dialogo fra una serva, Tarlesca, e l'imbroglione Bordegasso, che finisce per toglierle i due talleri che lei ha avuto dalle monache per comprare il pane. Caduta nella trappola 
di Bordegasso la povera Tarlesca saprà tirare la somma morale della sua esperienza:

Inscí resta ingannàe

Chi par trovà danè va sgiò da stràe.

Ho fàe, che no l'è poch se no m'impicch,

Inscí, per fà danè con sti mè cabel,

La maggior part diventa miserabel

Par l'ingodissa de diventa Richh.

(il lotto di Genova, vv. 499-504)

[Cosí resta ingannato

Chi per trovar denari esce di strada.

Cosí per far denari con questi miei intrighi,

Ho fatto che è già molto se non $\mathrm{m}^{\prime}$ impicco,

La maggior parte degli uomini diventa miserabile

Per l'ingordigia di diventare ricca.]

Ma l'intermezzo è soprattutto notevole per il modo in cui sottolinea i sogni dei poveri di migliorare con la fortuna la loro situazione economica disperata; anche se quasi sempre finivano vittime o della loro ossessione del gioco o dell'inganno di chi sapesse sfruttare l'ingenuità del povero ignorante.

Le 'bosinade' che parlano del lotto, come ci si aspetterebbe, non si contano. In una, "Dialegh tra 'l mari orb e soa miee lattiesta" [Dialogo tra il marito cieco e la moglie giocatrice], la moglie sogna di diventare ricca pure lei di colpo mentre il povero marito cerca di frenarla per evitare che l'ossessione che la domina la porti ad impegnare ogni loro avere per poter giocare ("Ma il gieuch l'ha d'ess poèu regolaa / In proporzione di not entra" [Ma il gioco va regolato / In proporzione ai nostri introiti]). ${ }^{63} \mathrm{E}$ in un' altra 'bosinada' Parpotera e Barlafusa dialogano sull' "Ingann di sogn, suppost bon per giugà al lott," cioè sull'inganno che è il lotto, che fa perdere chiunque ci giochi:

Adess capissi ch'hin bamban

E i sogn del lott in tutt ingan,

E con sti ball e con sti sogn

Chi giuga appont per el besogn

Ma ho semper osservaa

Che perden per la nezessitaa. ${ }^{64}$

[Adesso capisco che frottola

E i sogni della lotteria ingannano in tutto, 
E con queste balle e questi sogni

Quelli che giocano per bisogno

Ho sempre osservato

Che quelli perdono per necessità.]

mentre Balestrieri si concentra sul modo in cui il lotto pervade ogni livello della società:

Me suggeriss a proposit de quest

Anch i truccad che aveva lassaa foeura,

E sí che sti truccad comenzen prest,

Col trass coi provoch giò de post scoeurb,

In seguet poèu granc malizios a lest,

Per fann stà sott ne dan la gambiroeura

Guanci non san né fan alter mestee

Per avanzass che trà el compagn indree? ${ }^{65}$

[Mi viene in mente a proposito di questo

Anche i trucchi di cui non avevo prima parlato,

E sí che questi trucchi comincian presto

Fra quelli che lo fanno appena hanno lasciato la scuola,

Senza neanche avere scopo malizioso,

Fanno il gambetto per vincere

Spesso non sanno fare altro mestiere

Che quello d'avanzarsi ingannando i compagni.]

finendo in tipico tono settecentesco con un richiamo ad un regolamento piú stretto dei giochi di cui i poveri sono tanto spesso le vittime.

Le Osterie. L'ambiente che la 'bosinada' preferisce rappresentare in vena di svago è l'osteria, tradizionale centro della vita popolare milanese, ${ }^{66}$ cantata fin dal Lomazzo. Una 'bosinada' scritta sul fallimento d'un'osteria particolarmente frequentata, $\mathrm{Ca}^{\prime}$ Balena, ${ }^{67} \mathrm{fa}$ spia della serietà con cui il popolo prendeva questi posti in cui si passava gran parte del tempo libero.

Raro perciò lo scrittore erudito di dialetto che non avesse qualcosa da dire a riguardo. Maggi, nel Barone di Birbanza, aveva fatto recitare tutto un elenco di quei luoghi da Meneghino, il quale, pensando di dovere abbandonare la sua città prende congedo annoverando le piú famose osterie del tempo, dandoci cosí una buona guida gastronomica di Milano del tardo Seicento. Lo stretto rapporto fra osterie ed amore della città nella mente del popolano era chiaro per Maggi: 
Chaer el me chaer Miran, me creppa el coeur

$\mathrm{D}^{\prime}$ vett la bandonà

Se pú do's vedamm riveder

Mortadell di Tri Scagn

Busecca del Goeugga. . .

Moscatell d'i Tri Re. (BB, II, 3)

[Cara, oh mia cara Milano, mi crepa il cuore

Doverti abbandonare

Se non ci rivediamo addio

Mortadella di Tre Scagni

Busecca della Gobba

Moscatello dei Tre Re.]

Questo stesso rapporto lo capiva anche il Tanzi, "Viva pú l'osteria / Che l'è amno pú bon loeugh che al mond che sia!" [Viva pure l'osteria / Che non c'è luogo migliore al mondo!] il quale accenna in questa poesia anche ai 'Tri Re' menzionato da Maggi, e nei versi che seguono al valor sociale dell'osteria come luogo di svago e di sfogo per la classe popolare:

L'è on gran loeugh l'ostaria. Loeugh che l'è bon

E per tucc e per tutt. L'avegh passion

L'hoo anca lee per vertú

Chi almanc on pover omm el pò esalà

Lontan de' quell gran cruzi de sentí

La donna a tontonà.

[È un gran posto l'osteria. Luogo buono

Per tutto e per tutti. Ha passione,

Ma per me ha anche le sue virtú

Anche il povero può andarci a prender un po' d'aria

Lontano dai crucci della vita,

Dai borbottii della moglie.]

e chiude con un riferimento a Meneghino e all'eloquenza che Baltramina aveva detto in Concorso che i candidati dovevano trovare nelle cose:

Me maravej de noeuv; e el m'e duvis 
Che Meneghin el podess anch savè

Che a mesura del meret e del giust,

El galantomm el loda

I coss, o je desloda

Adess se me svoja el goss

Lodand a spada tratta l'osteria. ${ }^{68}$

[Mi meraviglio ancora e mi pare

Che Meneghino potesse anche saperlo

Che a misura del merito e del giusto,

Il gentiluomo loda

le cose o le critica

Adesso mi sgolo

A lodare a spada tratta l'osteria.]

Mi pare che, in modo particolare, questa breve rassegna di alcuni dei temi di comune popolarità sia nella 'bosinada' che nella poesia erudita dialettale ci dia altra prova del reciproco influsso fra i due generi nel loro sviluppo fino all'Ottocento, cioè che la 'bosinada' è stata man mano assimilata ed elevata dai poeti eruditi di dialetto fin dal Lomazzo, i quali però sono rimasti sempre ligi al suo spirito popolare. Abbiamo seguito l'evolversi del personaggio del 'bosin' fino alla sua identificazione con il poeta dialettale erudito e abbiamo potuto costatare un'ampia comune ispirazione tematica. La scarsa considerazione critica in cui s'è tenuta la 'bosinada' non può perciò sminuirne l'inciso storico-letterario, né ci può indurre a non riconoscere le reali tappe in cui il genere si sviluppò.

Per concludere, possiamo ritornare al punto da cui avevamo preso l'avvio e intravedere fin da questo momento, brevemente, la presenza della 'bosinada' in una opera di Carlo Porta. Ai tempi in cui Cherubini fece la sua raccolta di poesie dialettali, uno degli esemplari inclusi era il "Brindes de meneghin a l'ostaria" del Porta, allora l'unico esempio di poesia ditirambica in dialetto. ${ }^{69}$ In uno stile che ricorda quello di Redi di Bacco in Toscana, Porta segue l'ubriacarsi progressivo del narratore con parole che man mano assumono tonalità onomatopeica. La voce che narra è quella d'un 'Meneghino'/'bosin':

Vin nostran, vin di noster campagna

Ma leggitim, ma s'cett, ma sinzer,

Par al stomegh d'on bon Milanes

Che va roba del noster paes. ${ }^{70}$ 
[Vino nostrano, vino della nostra campagna.

Ma genuino, ma schietto, ma sincero,

Per la pancia d'un buon milanese

Ecco roba del nostro paese.]

E questa voce che parla dell'osteria dei buoni vini della sua città, Milano, è a nostro avviso la stessa che si trova in Lomazzo, Arese, Maggi, Tanzi, ecc. È anche, com'era mia intenzione documentare fin qui - nonostante l'ovvia superiorità metrica portiana - quella stessa che nasce nella 'bosinada.' Accettando questa teoria, una rivalutazione di questo genere come fonte della letteratura milanese / lombarda, almeno per quanto riguarda gli elementi tradizionalmente caratteristici d'essa, sembrerebbe storicamente e letterariamente piú che giustificata.

\section{University of Missouri at Columbia}

\section{NOTE}

1 Milano, Biblioteteca Nazionale Braidense, Sala Foscoliana 167.2/5.

2 Francesco Cherubini, Vocabolario Milanese-Italiano, I (Imp. Regia Stamperia, $1839)$, p. 140, dove dice inoltre "La maggior parte sono scritte male; ma non pertanto s'hanno il pregio cosí di diffondere la buona morale fra il popolo, come di far vivo ritratto delle mutazioni che d'età in età va sopportando il dialetto, e di consumare memoria delle costumanze e degli aneddoti del paese."

3 Ibid.

4 Infatti una delle prime 'bosinade' scritte alla fine del Cinquecento da Lomazzo satirizza le gride.

5 Gabrielle Fantuzzi, Letteratura Italiana, III: I Minori (Milano: Carlo Marzorati, 1962), pp. 2293-325. Nel suo saggio su Carlo Porta, Fantuzzi propone la tesi che la classificazione del Porta come 'minore' sarebbe in misura rilevante dovuta all'associazione dello scrittore al folclore dialettale. La responsabilità maggiore circa questa riduzione sarebbe, secondo Fantuzzi, del Cherubini, il quale, nel suo vocabolario di dialetto milanese aveva nominato Porta come autore apprezzato di 'bosinade,' che anch'egli riteneva unicamente forma folcloristica.

6 Ibid., p. 2296.

7 Bernardino Biondelli, Saggi sui dialetti gallo-italici (Milano: Bernardoni di Gio., 1853), p. 97.

8 Per 'letteratura erudita dialettale' intendo quella letteratura d'impegno e firmata che si sviluppava insieme a quella anonima.

9 Filippo Fichera, Mimi popolari lombardi (le bosinade) (Milano: Edizioni del Convivio letterario, 1963).

10 Ibid., p. XIV.

11 Ibid., p. XXIII.

12 Ho usato la versione riportata da L. Fichera, ma questa 'bosinada' si trova attualmente anche nella Biblioteca Ambrosiana con la segnatura SN VII 13.

13 Milano, Biblioteca Ambrosiana, Raccolta SC VII 1/43. Un'altra raccolta riporta 'bosinade' sull'assedio del Castello Sforzesco da parte degli austriaci il 23 maggio 1799 e sull'eclisse del sole avvenuto l'11 febbraio 1804 (SC VII 13).

14 Biblioteca Ambrosiana, SC VI 8/4. 
$15 \mathrm{Mi}$ sono servito dei vari articoli componenti questo studio che prima apparvero sull'Archivio Storico Lombardo: Giovanni De Castro, "La Storia nella poesia popolare milanese," ASL, Serie I, Vol. IV, 483-526 e 795-839; Vol. V, 223-55; Vol. Vl, 84-108.

16 Paolo Nalli, "Saggio di una bibliografia delle 'bosinade'," Rivista italiana di letteratura dialettale (Primo e secondo Trimestre 1937), 1-6.

17 A.M. Pizzagalli, "Carlo Maria Maggi e la vita milanese del '600," Rivista di letteratura dialettale VI (1931), 96-102.

18 I componimenti in questione, elencati e discussi da De Castro ma non reperibili oggi, sono i seguenti:

Filauro - Solacciosa commedia d'un solo atto, senza distinzione di scene, di vario metro e mescolata di molto linguaggio lombardo, in casa di maestro Girolamo di Benedetti, Bologna, 1520.

Il Muratore - Commedia rusticale lombarda. Siena, G. De Alessandro libraro, 1551.

Tonio e Pipo il Contadino e l'oste - Commedia in dialetto lombardo, senza anno di pubblicazione ma che De Castro ritiene del Cinquecento. op. cit. ASL, Vol. V. 243-4.

Cherubini, in Alcune delle migliori opere scritte in dialetto milanese (Milano: Pirotta, 1816), cita la Farsa del Bracho e del Milanese innamorato in Ast nel libro Opera No. D. Johannis Georgii Alioni Astensis, metro macharonico materno e gallico composita (Impressum Ast per magistrum Francischum de Silva, anno domini milesimo quingentesimo vigesimo primo, die ximensis martii). La farsa, scritta parte in astigiano parte in milanese, sarebbe forse la prima scrittura in milanese, e Cherubini ne cita quanto segue per dar un'idea del dialetto di allora:

Vada a Meren chi vol guadagn

E bon marchaa. Vu haury lasagn

Piena scudella al bon comijn,

$\mathrm{Cu}$ del formag piú dun sesin.

El dan mo lor per cincqu imbie, ecc., Cherubini, Vol. I, p. XXXIX.

Anche Biondelli cita quest'opera, associandosi all'opinione che sia la prima opera letteraria ad avere testo scritto in milanese: cit. p. 111.

19 Gianpaolo Lomazzo, Rabisch (Milano: Bidelli, 1627). La prima edizione di quest'opera fu stampata nel 1589.

20 Cherubini porta alcuni nomi degli autori anonimi piú conosciuti: Bernardo Rainoldi, Girolamo Maderna, Scipione Delfioni, Petrasanta, Domenico Francolini, Paolo Majnati, Giuseppe Abbiati e Gaspare Fumagalli: Cherubini, Vocabolario Milanese-Italiano, p. 140.

21 Devo avvertire il lettore che le 'bosinade,' anche quelle firmate, furono spesso stampate e/o trascritte con molti errori.

22 Citato dal Cherubini, Alcune delle migliori opere, Vol. IV, p. 245.

23 L'Accademia di Blenio diventò dopo quarant'anni la Gran Badia doi Facquin del Lag Mejó, indicando nel suo nome, secondo De Castro, di essere un'imitazione d'una vecchia società di facchini che andavano in giro a festeggiare il giorno del patrono, suonando pive, ecc., adoperando costumi simili a quello dal servo Meneghino del Maggi, e partecipando a diverse occasioni di pubblica allegria, specialmente ai carnevali, onde l'associazione della 'bosinada' col carnevale, tema che essa tratta spesso. De Castro, op. cit., Vol. VI, p. 84-5.

24 Curiosamente perfino Fantuzzi, nei suoi commenti riguardanti la traduzione di Porta della Divina Commedia in milanese, insiste su queste stesse caratteristiche come tipiche dell'autore che egli ritiene si stacchi in tutto dalla tradizione "folcloristica" della 'bosinada': 
Il popolano viaggiatore dell'aldilà non è il politico attivo e partecipe della vita pubblica: è una sorte di Renzo-Giovannin Bongèe in vena di raccontare al popolo la propria straordinaria e terrificante avventura.

Piú giú citando Isella, accenna agli "umori comico-realistici" dell'opera che portano alla "osservazione diretta di una realtà contemporanea e una prima caratterizzazione di una psicologia da popolano." Gariele Fantuzzi, "Carlo Porta," Letteratura Italiana: I Minori, già cit., p. 2300. In modo piú globale, Sapegno, risalendo alle origini, e parlando dei testi ducenteschi di Uguccione da Lodi, Pietro Bascapè ed altri, dice:

In tutti questi, non escluse le imitazioni degli enuegs occitanici, si riflette un gusto di concretezza, un'aderenza ai motivi di costume e di vita quotidiana, un proposito di riflessione etica e di pratica esortazione, che non è soltanto imposto dai modelli forestieri, bensí risponde a un bisogno intimo, e in cui comincia a predere forma la vocazione realistica che contrassegnerà nel tempo la civiltà. Walter Binni e Natalino Sapegno, Storia Letteraria delle Regioni d'Italia, "Lombardia" (Firenze: Sansoni, 1968), p. 64.

25 De Castro, op. cit., Vol. IV, 500.

26 Ibid., 501 ss., dove parla di un protagonista storico tipo Ruzzante, ma di tutti i tempi, il contadino vittima immediata delle stragi e delle battaglie successe in seguito alle guerre delle grandi potenze lontane e al contadino sconosciute.

27 Il Rabisch riporta altri nomi tipici dei 'bosin' che rimarranno nella cultura della 'bosinada.' Vedasi ad esempio p. 10, "Sor Medem," novella composta da "Barba Rognazzo," evidente nome accademico, e una 'bosinada' del 1814 citata da Nalli "L'è Rogn quel gran om, ch'el ch'el cerca tra tanti on galantom." (Nalli, cit., p. 23, No. 39), oppure nella poesia dello stesso Maggi "Canzone morale inferita da un ortolano" in cui menziona il "compagn Togn."

28 Lomazzo, Rabisch, "Don Compà Bornin gran Scancierè vell del Bregn," p. 7.

29 Pizzagalli, cit., 5.

30 De Castro, cit., VI, 86.

31 Il sonetto continua:

Per tí Porta Comasna e Porta Snes

Far Parí la Dovana ona gasciotta:

La busecca no sa quell che s'barbotta

Sui toeu Sonitt tant inscí ben desten

Tal che te po peccià vun de sti dí

Ch'el popel ha dezis de fatt onor,

A mett la bria e il bast al Pegasell,

E vení coi pavitta e tutta la brigaa

La incoronatt de verz e cervellaa.

32 Vedasi al riguardo un mio saggio, "Carlo Maria Maggi and the Bosinada," Forum Italicum, $13 / 4$ (1979), 480-95.

33 Cosí la definizione del Biondelli:

Questo nuovo eroe della musa lombarda era un servo fedele, ammogliato, carico di figli, ingenuo, faceto ed arguto, timido e franco ad un tempo, d'ottimo cuore e vittima sempre dei piú scaltri. Op. cit., p. 98.

34 Riporto la versione di Biondelli, pp. 115-16.

35 Per tutte le citazioni delle commedie di Maggi uso l'edizione a cura di Dante 1sella: Carlo Maria Maggi, Il Teatro Milanese, a cura di Dante Isella (Milano: Giulio Einaudi Editore, 1964).

36 Girolamo Birago, n. Milano $1691 \mathrm{~m}$. ivi 1773. Ricco come l'era stato anche Maggi, ed aristocratico, nel 1713 divenne Lettore di Logica alle scuole Cannobiane e Vicario del Podestà. Fu tre volte Giudice Pretorio. (Vedansi le note biografiche di Cherubini, Alcune delle migliori opere, Vol. I, p. 9). 
37 Questa commedia fu stampata nel 1724 a Milano da Francesco Nora. Birago scrisse pure una poesia dedicata a Meneghino, "Meneghin a la Sanaira. Testament de Meneghin."

38 Nato alla fine del Seicento a Milano, morto ivi nel 1755. Fece, come Maggi, carriera di impieghi pubblici, e nel 1713 fu nominato sopranumerario di governo. Cherubini, cit., Vol. IV, pp. 210-11.

39 Ibid., p. 229.

40 Nato a Milano alla fine del Seicento, morto ivi 1754, di famiglia liberale. Percorse la carriera ecclesiastica.

41 Cherubini, Opere, cit., Vol. IV, p. 245.

42 Carlo Tanzi, Milano, 1700-62.

43 Domenico Balestrieri, 1714-18 (Milano), educato in retorica alle Arcimbolde, in filosofia poi dai gesuiti di Brera e attivissimo nell'accademia dei Trasformati. Fra le molte opere spiccano Rimm Milaness (1774), una traduzione in Milanese della Gerusalemme Liberata pubblicata dopo 16 anni di lavoro nel 1772, e Il figliol prodigo, 1778.

44 Cherubini, Opere, cit., Vol. V, pp. 348-55.

45 Balestrieri, in Cherubini, Opere, cit., Vol. V, p. 306.

46 Balestrieri, Il figliol prodigo (Milano: Marelli, 1748).

47 Balestrieri, "La Badia di Meneghi." A consulta sora el Dialegh de la lengua Toscana (Milano: Agnelli, 1759).

48 Ibid., p. 46.

49 Prima versione pubblicata da Giovanin Giacomo a Como nel 1606, è una spiegazione delle origini del dialetto con varianti e piccolo vocabolario.

50 Balestrieri, La Badia, cit., pp. 68-70. Inoltre, alla pagina 69 esalta la tradizione maggesca per avere sublimato i valori popolari in dialetto:

$\mathrm{E}$ in del concors di noster Meneghitt

$\mathrm{O}$ in bocca de Tarlesca

O pú de Beltramminna

Pasen dalla cantina alla baltresca.

Fa lo stesso uno dei poeti minori del Settecento, Carl'Andrè Oltolinache, che scrisse di Baltramina ancora come musa, nella seconda metà del secolo:

Nú Beltramina musa, che bescanta

Sui rivi de la Vecchiabbia, n'hoo pragura

Che la mia poesia bella e frizzanta

No possa fà anca lee la soa figura,

Che soo famm anch coi verz on poo d'onor

Col faj passà in Parnass per cavolfior.

(Vedasi Cherubini, Opere, cit., Vol. IX, pp. 69-75)

51 Biondelli, cit., p. 97, lo chiama il padre e fondatore della poesia milanese. De Castro lo definisce "mediocre" (cit., Vol. V, 251) e Cherubini include nella sua raccolta alcune delle poesie di Lomazzo "non già per merito distinto che esse abbiano in sé stesse, ma bensí per dare a conoscere le prime poesie che siano state scritte in dialetto milanese," Vol. I, p. 6.

52 Uso la versione riportata da Cherubini, Opere, cit., Vol. I.

53 De Castro, cit., Vol. VI, 98-105.

54 lbid., 105.

55 Ibid.

56 Ibid.

57 Vedasi ad esempio l'intermezzo de Il falso filosofo.

58 Vedasi il secondo intermezzo de I consigli di Meneghino sopra i diversi modi di ridere, buon esempio del tema 'ridicoloso.'

59 Milano, Biblioteca Ambrosiana, Racc. Bosinaa SLD I. 5588.

60 Milano, Biblioteca Ambrosiana, Racc. Bosinade SCV II 1/18. 
61 Carlo Tanzi, "Sora la Spilorciaria," in Cherubini, Opere, cit., Vol. IV, pp. 324-30.

62 Biblioteca Braidense, Sala Foscoliana, V. 167. 2/40 (1812).

63 Biblioteca Braidense, Sala Foscoliana, V. 167. 1/32.

64 Ibid.

65 Balestrieri, "Per on'Accademina sora i goeugh," in Cherubini, Opere, cit., Vol. VI, pp. 48-54.

66 Questa, nella biblioteca Braidense (Sala Foscoliana V. 167. 2/59), è una delle piú tipiche:

Allegrament o cittadin

Per divertivv l'è chi el Bosin,

Con una voeura Bosinada

Sul costum invetraa

De quij Bottiatt e operai

Che tutt quant, quel poc salari

El van tutt l'ann a butta via

In dí di festa all'ostaria.

67 Biblioteca Braidense, Sala Foscoliana V. 167. 1/56.

Allegrament na Bosinada

Per divertí chi la brigada

Cantem on poo su on argoment

Che già san tutta la gent

E che paricc veden con pena.

Sul fallliment de ca Balena.

68 Carlo Tanzi, "Recitaa in l'Accademia sora l'ostaria" in Cherubini, Opere, cit., Vol. IV, pp. 350-55.

69 Carlo Porta, "Brindes de Meneghin a l'Ostaria per l'entrata in Milan de S.M. l'Imperator Franzesch prim in compagnia de Soa Miee l'Imperatriz Maria Luisa," in Cherubini, Opere, cit., V. XII, pp. 162-72.

70 Ibid., p. 166. 